\title{
AVALIAÇÃO DO DESGALHADOR FLORESTAL DE DISCOS EM FUNÇÃO DO TEMPO DE ESTOCAGEM DA MADEIRA EM CAMPO
}

\author{
ASSESSMENT OF FOREST DELIMBER IN FUNCTION OF TIME WOOD STORAGE IN \\ FIELD
}

\author{
Rodrigo Petrongari Tonin ${ }^{1}$ Ricardo Hideaki Miyajima ${ }^{1}$ José Raimundo de Souza Passos $^{2}$ Paulo Torres \\ Fenner $^{3}$
}

\section{RESUMO}

Com as inovações da colheita mecanizada no Brasil, tornam-se indispensáveis estudos para obtenção de dados reais sobre as novas máquinas que estão surgindo no mercado. O estudo objetivou a avaliação técnica e de custos de um desgalhador florestal de discos em árvores com diferentes tempos de estocagem em campo, e verificar possíveis correlações entre umidade da madeira, produtividade e custo de produção. Para a análise técnica foi realizado um estudo de tempos e movimentos pelo método de cronometragem de tempos contínuos e para a análise de custos, calcularam-se os custos operacionais através do método proposto pela ASABE. Aproximadamente, aos 40 dias em campo, as árvores atingiram a umidade de equilíbrio, o teor de umidade não demonstrou possuir correlação com o rendimento da máquina, que foi na média $643,34 \mathrm{~m}^{3} / \mathrm{h}$, e nem com o custo de produção, sendo, o custo operacional de R \$180,04/hora, e o custo de produção médio $\mathrm{R} \$ 0,28 / \mathrm{m}^{3}$.

Palavras-chave: colheita de madeira; desgalhamento; produtividade; custos.

\begin{abstract}
With the innovations of mechanized harvesting in Brazil, become necessary studies to obtain real data on the new machines that are emerging in the market. The study aimed to the technical evaluation and cost a forest delimber on trees with different times storage in the field, and verify possible correlations among wood moisture, productivity and cost of production. For the technical analysis was conducted a study of time and motion by timing method of continuous time and for the cost analysis, we calculated operating costs through the method proposed by ASABE. Approximately 40 days in the field trees reached the equilibrium moisture content, the moisture has demonstrated no correlation of content with the performance of the machine, which was on average $643.34 \mathrm{~m}^{3} / \mathrm{h}$, and not with the cost of production, and, the operating cost of $\mathrm{R} \$ 180.04$ / hour, and the production cost average $\mathrm{R} \$ 0.28 / \mathrm{m}^{3}$.
\end{abstract}

Keywords: timber harvesting; delimbing; productivity; costs.

\section{INTRODUÇÃO}

No setor do agronegócio brasileiro, a atividade florestal detém lugar de destaque, em 2013, o setor brasileiro de árvores plantadas empregou diretamente em torno de 630 mil pessoas e adicionou ao Produto Interno Bruto (PIB) cerca de R 56 bilhões, representando 1,2\% de toda a riqueza gerada no País (INDUS-

1 Engenheiro Florestal, MSc., Doutorando em Ciência Florestal, Faculdade de Ciências Agronômicas, Universidade Estadual Paulista Júlio de Mesquita Filho, Rua José Barbosa de Barros, 1780, Jardim Paraíso, CEP 18610-307, Botucatu (SP), Brasil.rp.tonin@hotmail.com / richidetoshimiyajima@hotmail.com

2 Engenheiro Florestal, Dr., Professor Assistente do Departamento de Bioestatística, Instituto de Biociências, Universidade Estadual Paulista Júlio de Mesquita Filho, Rua Prof. Dr. Antônio Celso Wagner Zanin, 250, Distrito de Rubião Junior, CEP 18618-689, Botucatu (SP), Brasil. jrpassos@ibb.unesp.br

3 Engenheiro Florestal, Dr., Professor Adjunto do Departamento de Ciência Florestal, Faculdade de Ciências Agronômicas, Universidade Estadual Paulista Júlio de Mesquita Filho, Rua José Barbosa de Barros, 1780, Jardim Paraíso, CEP 18610-307, Botucatu (SP), Brasil. fenner@fca.unesp.br 
TRIA BRASILEIRA DE ÁRVORES, 2014).

Nos últimos anos, o setor florestal brasileiro despontou rapidamente graças aos avanços da mecanização da colheita e do transporte de madeira (YONEZAWA, 2010).

A evolução da mecanização nas operações de colheita de madeira é motivada pela necessidade de melhoria das condições de trabalho, redução da mão de obra, aumento da competitividade com o incremento de novas alternativas de produção (SEIXAS, 2010).

A intensa mecanização desencadeou um processo contínuo de avaliação dos rendimentos operacionais e dos custos, devido à colheita representar um percentual superior a $50 \%$ do total dos custos finais da madeira posta na indústria (SIMÕES, 2008; MACHADO, 2014).

Dada a grande quantidade de máquinas e equipamentos de corte e extração disponíveis no mercado, as empresas podem formar vários conjuntos de colheita que podem ser empregados, cabendo a cada empresa optar por aquele que seja mais adequado ao sistema utilizado e às suas peculiaridades (JACOVINE et al., 2005).

Em alguns sistemas é possível a realização da operação de desgalhamento das árvores (WALDRIGUES, 1983).

Segundo Sant'ana (2014), existem três maneiras de realizar o desgalhamento das árvores: manual, semimecanizado e mecanizado. O manual é realizado com uso de ferramentas como: machado, foice, facão, serra de arco. O semimecanizado é realizado com o uso de motosserras ou motopodas, equipamentos que permitem mecanizar parte da atividade, porém, ainda com grande esforço físico dos trabalhadores. $\mathrm{O}$ mecanizado é realizado com o uso de máquinas e equipamentos, como o Harvester, Stroke Delimber, que são máquinas que possuem melhor rendimento e oferecem maior conforto e segurança aos operadores.

Criado pela equipe da gerência de Colheita da Aperam Bioenergia, o desgalhador florestal de discos promete trazer a operação de desgalhamento para um novo patamar, oferecendo maior segurança e rendimento operacional (SOCIEDADE DE INVESTIGAÇÕES FLORESTAIS, 2014). Este equipamento possui um implemento com discos planos recortados que pode ser acoplado em Skidders ou em outros modelos de máquinas-base (INSTITUDO DE PESQUISA E ESTUDOS FLORESTAIS, 2012).

Este trabalho teve como objetivo geral realizar uma análise técnica e de custo do desgalhador florestal de discos. Os objetivos específicos foram: determinar a produtividade e os custos de produção em função do período de estocagem da madeira em campo.

\section{MATERIAL E MÉTODOS}

\section{Caracterização da área de estudo}

Esta pesquisa foi realizada entre os meses de janeiro e fevereiro de 2014 em uma empresa florestal localizada no município Itatinga, Estado de São Paulo, com coordenadas geográficas Latitude: $23^{\circ} 6$ '9" S e Longitude: $48^{\circ} 36^{\prime} 55^{\prime}$ 'W, com altitude de 826,2 metros.

O clima característico da região segundo a Köppen e Geiger é classificado como Cfa, com concentração das chuvas no verão e seca no inverno. Pluviosidade média anual de $1308 \mathrm{~mm}$ e temperatura média anual de $19.2^{\circ} \mathrm{C}$.

O relevo da região de estudo era plano e o experimento foi instalado em uma floresta homogênea de Eucalyptus grandis, clonal, de primeiro corte, com sete anos de idade.

Foi realizado o inventário do talhão no qual foi alocada a área experimental, o que possibilitou identificar que havia 1333 árvores por hectare, com (diâmetro a altura do peito) DAP médio de $15 \mathrm{~cm}$, altura média do povoamento de $25,4 \mathrm{~m}$ e volume médio individual de $0,21 \mathrm{~m}^{3}$.

Após a derrubada das árvores e posterior arraste para a margem do talhão, a parcela foi demarcada com dimensões de $80 \mathrm{~m}$ de largura e comprimento equivalente à altura das árvores, em média $25 \mathrm{~m}$, totalizando uma área de $2000 \mathrm{~m}^{2}$.

\section{Tratamentos estudados}

Foram instalados na área experimental cinco tratamentos, que se diferenciavam pelo tempo de es- 
tocagem da madeira em campo antes da realização do desgalhamento.

- Tratamento 1: árvores permaneceram 10 dias em campo antes de serem desgalhadas, sendo 5 dias no interior do talhão e 5 dias na bordadura.

- Tratamento 2: árvores permaneceram 20 dias em campo antes de serem desgalhadas, sendo 5 dias no interior do talhão e 15 dias na bordadura.

- Tratamento 3: árvores permaneceram 30 dias em campo antes de serem desgalhadas, sendo 5 dias no interior do talhão e 25 dias na bordadura.

- Tratamento 4: árvores permaneceram 40 dias em campo antes de serem desgalhadas, sendo 5 dias no interior do talhão e 35 dias na bordadura.

- Tratamento 5: árvores permaneceram 50 dias em campo antes de serem desgalhadas, sendo 5 dias no interior do talhão e 45 dias na bordadura.

Cada tratamento possuía 16 metros de largura e aproximadamente 25 metros de comprimento, totalizando uma área de aproximadamente $400 \mathrm{~m}^{2}$. Os tratamentos foram subdivididos em quatro eitos de trabalho de quatro metros de largura cada (Figura 1).

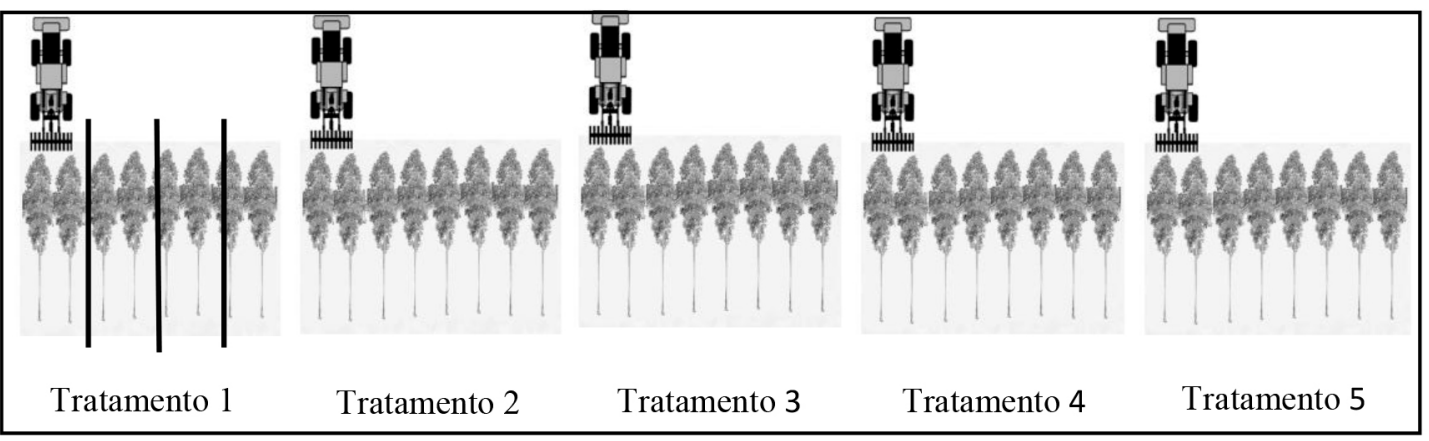

FIGURA 1: Croqui da área experimental.

FIGURE 1: Sketch of the experimental area.

\section{Equipamento e atividade estudada}

O desgalhador florestal de discos é um equipamento com função de realizar o desgalhamento das árvores. É composto por uma máquina-base sem a estrutura da pá carregadora, com rodados de pneus, equipado com um implemento acoplado ao braço hidráulico, composto por discos planos recortados.

O desgalhamento ocorria com as árvores dispostas na bordadura do talhão. Após serem arrastadas pelo Skidder, a máquina se deslocava longitudinalmente aos fustes para realizar a operação.

\section{Ciclo operacional da máquina}

As operações do Desgalhador Florestal de Discos foram divididas em atividades parciais, e estas classificadas em atividades efetivas (AE) e atividades gerais (AG).

As atividades parciais efetivas do desgalhador florestal de discos foram:

- Deslocamento (DL): tempo despendido em que a máquina se movimenta sobre a copa das árvores com o implemento erguido, sendo finalizado quando o implemento era abaixado para iniciar a operação de desgalhamento.

- Desgalhamento (DG): tempo despendido para efetuar o desgalhamento das árvores, movimento de marcha à ré. Iniciava-se quando o implemento encostava os discos nas árvores derrubadas e era finalizado quando o implemento era elevado e perdia o contato com as árvores.

Já as atividades parciais gerais do desgalhador florestal de discos foram:

- Manutenção: tempo despendido para realização de manutenção da máquina ou do implemento florestal.

- Paradas pessoais: tempo despendido para necessidades fisiológicas do operador. 


\section{Análise técnica}

Foi realizado um estudo de tempos e movimentos pela cronometragem de tempo contínuo, que consiste em realizar as medições sem que o cronômetro seja parado.

\section{Rendimento operacional do desgalhador florestal de discos}

O rendimento operacional do desgalhador florestal de discos foi obtido por meio do volume de madeira com casca $\left(\mathrm{m}^{3}\right)$ e do tempo efetivo consumido para realização da operação de desgalhamento.

$\mathrm{O}$ volume da madeira desgalhada foi obtido pela multiplicação do número de árvores desgalhadas em cada tratamento, pois foi contado o número exato de árvores que cada tratamento continha, pelo volume médio por árvore, sendo o volume médio das árvores obtido através do inventário florestal realizado.

O cálculo do rendimento operacional está representado na equação.

$$
R=v / t
$$

Em que: $\mathrm{R}=$ rendimento operacional médio $\left(\mathrm{m}^{3} / \mathrm{h}\right) ; \mathrm{v}=$ volume de madeira com casca desgalhada $\left(\mathrm{m}^{3}\right) ; \mathrm{t}=$ tempo $(\mathrm{h})$.

\section{Análise de custo}

A análise de custo foi realizada pela determinação do custo operacional e de produção do desgalhador na operação de desgalhamento. Para o cálculo do custo operacional utilizou-se a metodologia proposta pela American Society of Agricultural and Biological Engineers (ASABE) (AMERICAN SOCIETY OF AGRICULTURAL ENGINEERS, 2001) para as condições deste estudo. O custo operacional em cada tratamento foi estimado em reais por hora e custo de produção em reais por metro cúbico $\left(\mathrm{R} \$ / \mathrm{m}^{3}\right)$.

Considerando os custos fixos (depreciação, juros, abrigo, taxas e seguros) e os custos variáveis (combustíveis, reparos e manutenção, lubrificantes, mão de obra).

\section{Estimativas do custo de desgalhamento}

A estimativa do custo da colheita florestal $\left(\mathrm{R} \$ / \mathrm{m}^{3}\right)$ foi obtida conforme a equação.

$$
\mathrm{Ccm}=\mathrm{CopMF} / \mathrm{R}
$$

Em que: $\mathrm{Ccm}=$ custo da colheita da madeira com casca $\left(\mathrm{R} \$ / \mathrm{m}^{3}\right) ;$ CopMF $=$ custo operacional da máquina florestal $(\mathrm{R} \$ \mathrm{~h}) ; \mathrm{R}=$ rendimento $\left(\mathrm{m}^{3} / \mathrm{h}\right)$.

\section{Aferição da umidade da madeira nos tratamentos}

Para aferição da umidade da madeira, base seca, foram coletados três discos de quatro árvores de cada tratamento, um de cada eito de trabalho. Os discos foram pesados e posteriormente secos em estufa a $103^{\circ} \mathrm{C}\left(+\mathrm{ou}-2^{\circ} \mathrm{C}\right)$, e a umidade foi determinada pela equação.

$$
U=(P i-P s / P s) .100
$$

Em que: $\mathrm{U}=$ teor de umidade da madeira (\%); $\mathrm{Pi}=$ peso inicial (g); Ps = peso seco (g).

Assim foi possível definir o teor de umidade em cada tratamento e alguma possível correlação com a operação de desgalhamento.

\section{Análise estatística}

$\mathrm{Na}$ análise estatística das variáveis, rendimento $\left(\mathrm{m}^{3} / \mathrm{h}\right)$, custo $\left(\mathrm{R} \$ / \mathrm{m}^{3}\right)$ e umidade $(\%)$ foram utilizados modelos lineares generalizados com distribuição de probabilidade gama e função de ligação logarítmica (NELDER; WEDDERBURN, 1972; DIGGLE et al., 2002), considerando-se o tempo (dias) como fator. A qualidade do ajuste dos modelos foi feita através da análise de desvios (deviance). Para comparações entre 
os tempos foi utilizado foi o teste LSMeans do procedimento genmod do programa SAS (SAS INSTITUTE, 2012). E foi realizada correlação entre as variáveis, umidade $(\%)$, rendimento $\left(\mathrm{m}^{3} / \mathrm{h}\right)$ e custo $\left(\mathrm{R} \$ / \mathrm{m}^{3}\right)$.

\section{RESULTADOS E DISCUSSÃO}

\section{Teor de umidade da madeira}

O teor de umidade médio da madeira encontrado no talhão no qual o estudo foi realizado, logo após a derrubada das árvores, foi de $91 \%$, cujo valor diminuiu conforme o tempo de permanência das árvores no campo. As umidades de cada tratamento no momento do desgalhamento estão descritos na Tabela 1.

TABELA 1: Médias (desvio padrão entre parênteses) do teor de umidade da madeira (\%) em função do período de estocagem da madeira no campo.

TABLE 1: Mean (standard deviation in brackets) of the wood moisture content (\%) in function of time wood storage in field.

\begin{tabular}{cccccc}
\hline \multirow{2}{*}{ Variável } & \multicolumn{5}{c}{ Tratamentos } \\
\cline { 2 - 6 } & 1 & 2 & 3 & 4 & 5 \\
\hline \multirow{2}{*}{ Umidade } & $68,00 \mathrm{a}$ & $49,00 \mathrm{~b}$ & $42,00 \mathrm{c}$ & $38,00 \mathrm{~d}$ & $37,00 \mathrm{~d}$ \\
& $-0,82$ & $-1,41$ & $-0,82$ & $-1,41$ & $-1,41$ \\
\hline
\end{tabular}

Médias seguidas pela mesma letra na linha não diferem estatisticamente pelo LS Means ( $<<0,05)$.

Pela análise estatística verificou-se que somente os tratamentos 4 e 5 não diferem estatisticamente entre si, porque a umidade da madeira atingiu o equilíbrio aos 40 dias.

Observa-se que a maior perda de umidade ocorreu nos primeiros vinte dias de secagem, tempo semelhante ao relatado por Rezende et al. (2010) que trabalharam com secagem de toras de Eucalyptus ao ar livre.

Após 20 dias, a umidade da madeira foi reduzida em $46 \%$, valor próximo à redução de $50 \%$ relatada por Ponce e Watai (1985) e a encontrada por Rezende et al. (2010), que foi de $45 \%$.

A umidade de equilíbrio foi alcançada a partir dos 40 dias de estocagem, tempo próximo ao encontrado por Rezende et al. (2010), em que a umidade de equilíbrio foi alcançada a partir dos 50 dias.

\section{Análise técnica}

O tempo despendido para a operação do desgalhador florestal de discos foi composto unicamente de atividades efetivas, em todos os tratamentos.

$\mathrm{Na}$ Figura 2, são apresentados os percentuais dos tempos das atividades parciais que compõem o ciclo operacional do desgalhador florestal de discos. É possível verificar que a atividade parcial que despendeu maior tempo em todos os tratamentos foi "deslocamento", que na média representou 55,6\% do ciclo operacional. Enquanto a atividade parcial "desgalhamento" representou $44,4 \%$ do ciclo operacional. 


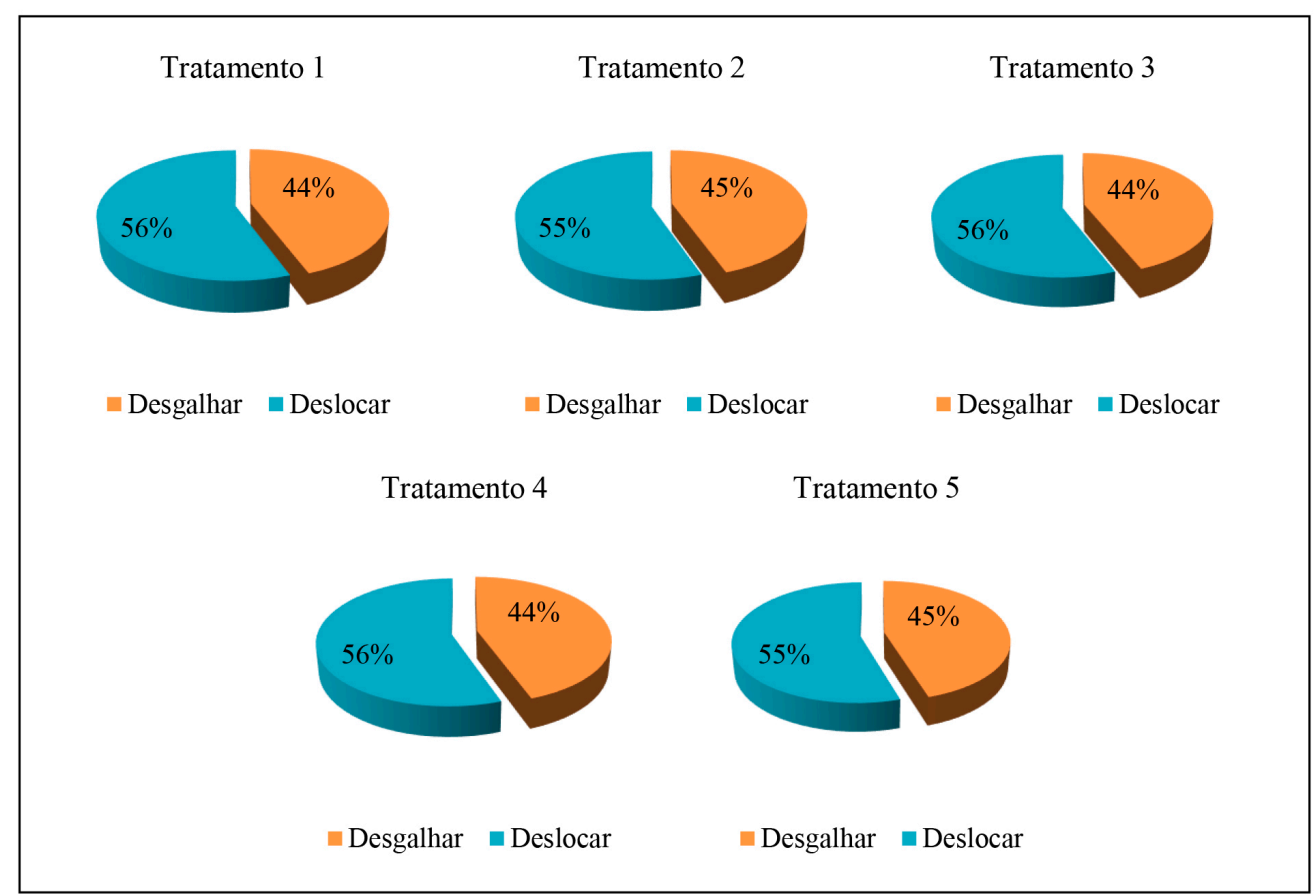

FIGURA 2: Tempos percentuais das atividades efetivas do desgalhamento nos tratamentos.

FIGURE 2: Percentage times of the effectives activities of the delimbing in treatments.

Apesar de ser um equipamento desenvolvido para efetuar o desgalhamento, a maior parte do tempo do ciclo operacional é utilizada com a operação de deslocamento, uma alternativa que pode ser estudada para reduzir o tempo de deslocamento, é no momento em que o desgalhador florestal de discos "sobe" nas copas das árvores, já abaixar o implemento e realizar o desgalhamento no movimento de frente e no de marcha à ré.

$\mathrm{Na}$ Tabela 2 são apresentados os rendimentos operacionais do desgalhador florestal de discos nos diferentes tratamentos. Os rendimentos operacionais efetivos do foram de $641,31 \mathrm{~m}^{3} /$ ha no tratamento 1 , $669,81 \mathrm{~m}^{3} /$ ha no tratamento $2,633,79 \mathrm{~m}^{3} /$ ha no tratamento $3,606,11 \mathrm{~m}^{3} /$ ha no tratamento $4 \mathrm{e} 657,22 \mathrm{~m}^{3} /$ ha no tratamento 5, com diferença estatística somente do tratamento 4 em relação aos demais.

TABELA 2: Médias (desvio padrão entre parênteses) do rendimento operacional $\left(\mathrm{m}^{3} / \mathrm{h}\right)$ do desgalhador florestal de discos em função do período de estocagem da madeira em campo.

TABLE 2: Mean (standard deviation in parentheses) operating income $\left(\mathrm{m}^{3} / \mathrm{h}\right)$ of forest delimber in function of time wood storage in field.

\begin{tabular}{cccccc}
\hline \multirow{2}{*}{ Variável } & \multicolumn{5}{c}{ Tratamentos } \\
\cline { 2 - 6 } & 1 & 2 & 3 & 4 & 5 \\
\hline \multirow{2}{*}{ Rendimento } & $649,31 \mathrm{a}$ & $669,81 \mathrm{a}$ & $633,79 \mathrm{ab}$ & $606,11 \mathrm{~b}$ & $657,22 \mathrm{a}$ \\
& $-27,34$ & $-34,74$ & $-37,58$ & $-14,4$ & $-34,84$ \\
\hline
\end{tabular}

Médias seguidas pela mesma letra na linha não diferem estatisticamente pelo LS Means ( $<<0,05)$.

O rendimento operacional médio do Desgalhador Florestal de Discos foi de $643,34 \mathrm{~m}^{3} \cdot \mathrm{h}^{-1}$, valor muito superior quando comparado ao desgalhamento efetuado com motosserra, sistema anteriormente utilizado pela empresa, que, segundo Seixas (1986), é de 23,07 $\mathrm{m}^{3} \cdot \mathrm{h}^{-1}$. 


\section{Análise de custos}

Na Tabela 3, são apresentados os valores dos custos fixos obtidos para o desgalhador florestal de discos.

Os custos fixos por hora do desgalhador florestal de discos totalizaram $\mathrm{R} \$ 53,55$.

TABELA 3: Custos fixos do desgalhador florestal de discos.

TABLE 3: Fixed costs of forest delimber.

\begin{tabular}{cc}
\hline Custos Fixos & $\mathrm{R} \$ / \mathrm{h}$ \\
\hline Juros & 11,55 \\
Depreciação & 37,8 \\
Abrigo & 1,58 \\
Taxas administrativas & 2,1 \\
Seguros & 0,52 \\
\hline Total & 53,55 \\
\hline
\end{tabular}

Os custos variáveis obtidos para o desgalhador florestal de discos totalizaram R $\$ 126,49$ por hora.

$\mathrm{Na}$ Tabela 4 podem ser observados detalhadamente os custos variáveis do desgalhador florestal de discos.

TABELA 4: Custos variáveis do desgalhador florestal de discos.

TABLE 4: Variables costs of forest delimber.

\begin{tabular}{cc}
\hline Custos Variáveis & $\mathrm{R} \$ / \mathrm{h}$ \\
\hline Mão de Obra & 35,16 \\
Combustível & 55 \\
Lubrificação & 0,41 \\
Reparos e manutenção & 35,91 \\
\hline Total & 126,49 \\
\hline
\end{tabular}

Diante do somatório dos custos fixos e variáveis, o desgalhador florestal de discos apresentou um custo operacional total de R $\$ 180,04$ por hora.

Na Tabela 5 são apresentados os custos de produção resultantes dos rendimentos operacionais efetivos desenvolvidos pelo desgalhador florestal de discos nos cinco tratamentos.

Verificou-se que, o custo operacional por hora foi de R\$180,04. Considerando a média dos rendimentos operacionais efetivos nos cinco tratamentos, o custo médio de produção resultou em $\mathrm{R} \$ 0,28$ por metro cúbico de madeira desgalhada. 
TABELA 5: Médias (desvio padrão entre parênteses) dos custos operacionais $\left(\mathrm{R} \$ / \mathrm{m}^{3}\right)$ do desgalhador florestal de discos em função do período de estocagem da madeira em campo.

TABLE 5: Average (standard deviation in parentheses) operating costs $\left(\mathrm{R} \$ / \mathrm{m}^{3}\right)$ of forest delimber in function of time wood storage in field.

\begin{tabular}{cccccc}
\hline \multirow{2}{*}{ Variável } & \multicolumn{5}{c}{ Tratamentos } \\
\cline { 2 - 6 } & 1 & 2 & 3 & 4 & 5 \\
\hline \multirow{2}{*}{ Custo } & $0,28 \mathrm{a}$ & $0,27 \mathrm{a}$ & $0,29 \mathrm{ab}$ & $0,30 \mathrm{~b}$ & $0,27 \mathrm{a}$ \\
& $-0,01$ & $-0,01$ & $-0,02$ & $-0,01$ & $-0,01$ \\
\hline
\end{tabular}

Médias seguidas pela mesma letra na linha não diferem estatisticamente pelo LS Means $(p<0,05)$.

\section{Correlação entre as variáveis}

$\mathrm{Na}$ Tabela 6 é possível verificar possíveis correlações das variáveis: teor de umidade da madeira rendimento, massa remanescente e custo.

TABELA 6: Matriz de correlação e (valor p entre parênteses) das variáveis: teor de umidade $(\%)$, rendimento $\left(\mathrm{m}^{3} / \mathrm{h}\right)$, massa remanescente $(\mathrm{kg} / \mathrm{ha})$ e custo $\left(\mathrm{R} \$ / \mathrm{m}^{3}\right)$.

TABLE 6: Correlation matrix and (p-value in parentheses) of the variables: moisture content (\%), yield $\left(\mathrm{m}^{3} / \mathrm{h}\right)$, remaining mass $(\mathrm{kg} / \mathrm{ha})$ and cost $\left(\mathrm{R} \$ / \mathrm{m}^{3}\right)$.

\begin{tabular}{cccc}
\hline & Umidade & Rendimento & Custo \\
\hline \multirow{2}{*}{ Umidade } & - & 0,201 & $-0,216$ \\
& & $-0,395$ & $-0,360$ \\
\hline \multirow{2}{*}{ Rendimento } & 0,201 & - & $-0,999$ \\
& $-0,395$ & & $-0,000$ \\
\hline \multirow{2}{*}{ Custo } & $-0,216$ & $-0,999$ & - \\
& $-0,360$ & $-0,000$ & - \\
\hline
\end{tabular}

$\mathrm{p}<0,05$ demonstram correlação entre variáveis.

A Tabela 6 demonstra que as únicas variáveis que possuem correlação são rendimento $\left(\mathrm{m}^{3} / \mathrm{h}\right)$ e custo $\left(\mathrm{R} \$ / \mathrm{m}^{3}\right)$, que apresentam o valor $\mathrm{p}$ menor que 0,05 .

\section{CONCLUSÕES}

O teor de umidade da madeira diminuiu com o aumento do tempo de estocagem das árvores em campo, atingindo a umidade de equilíbrio aos 40 dias.

Os rendimentos operacionais e os custos de produção do desgalhador florestal de discos não possuem correlação com o teor de umidade da madeira.

Os custos de produção aumentam com a diminuição do rendimento da máquina.

Neste estudo, o período ideal de estocagem em campo da madeira foi em torno dos 40 dias, quando a madeira atingiu a umidade de equilíbrio.

\section{REFERÊNCIAS}

AMERICAN SOCIETY OF AGRICULTURAL ENGINEERS. ASABE standards 2001: machinery, equipment, and buildings: operating costs. Iowa: Ames, 2001. 566 p. (ASAE D472-3).

DIGGLE, P. J. et al. Analysis of longitudinal data. 2nd ed. Oxford: Oxford University Press, 2002. 
INDUSTRIA BRASILEIRA DE ÁRVORES. BRACELPA. 2014. Disponível em: <http://www.bracelpa. org.br/shared/iba 2014 pt.pdf $>$. Acesso em: 12 jan. 2015.

INSTITUDO DE PESQUISA E ESTUDOS FLORESTAIS. IPEF Notícias. Piracicaba: IPEF, 2012.

JACOVINE, L. A. G. et al. Avaliação da perda de madeira em cinco subsistemas de colheita florestal. Revista Árvore, Viçosa, MG, v. 29, n. 3, p. 391-400, 2005.

MACHADO, C.C. et al. O setor florestal brasileiro e a colheita florestal. In. MACHADO, C.C. (Ed). Colheita florestal. 3 ed. Viçosa. UFV, 2014. cap. 1, p.15-45.

NELDER, J. A.; WEDDERBURN, R. W. Generalized linear models. Journal of the Royal Statistical Society Series A, London, v. 135, n. 3, p. 370-384, 1972. doi:10.2307/2344614.

PONCE, R. H.; WATAI, L. T. Manual de secagem da Madeira. Brasília: STI; IPT, 1985. 72 p.

REZENDE, R. N. et al. Secagem ao ar livre de toras de Eucalyptus grandis em Lavras, MG. Cerne, Lavras, v. 16, supl, p. 41-47, jul. 2010.

SANT’ANA, C. M. Corte. In: MACHADO, C. C. (Ed). Colheita Florestal. 3. ed. Viçosa, MG: UFV, 2014. p. 74-105.

SAS INSTITUTE. Statistical analysis system for Windows. release 9.2. Cary: SAS Inatitute, 2012.

SEIXAS, F. As inovações da colheita de madeira. Revista Opiniões, Ribeirão Preto, p. 38, jun./ago. 2010.

SEIXAS, F. Planejamento e estudo de sistema de exploração florestal. IPEF, Piracicaba, v. 34, p. 25-30, 1986.

SIMÕES, D. Avaliação econômica de dois sistemas de colheita florestal mecanizada de eucalipto. 2008. 105 f. Dissertação (Mestrado em Energia na Agricultura) - Universidade Estadual Paulista, Faculdade de Ciências Agronômicas, Botucatu, 2008.

SOCIEDADE DE INVESTIGAÇÕES FLORESTAIS. Aperam Bioenergia inclui desgalhador florestal em suas atividades. Minas Gerais: SIF, 2014. Disponível em: <http://www.sif.org.br/noticia/aperam-bioenergia-inclui-desgalhador-florestal-em-suas atividades>. Acesso em: 18 dez. 2014.

WALDRIGUES, O. M. P. Produtividade no Setor Florestal. 1983. 145 f. Monografia (Pós-Graduação em Engenharia Florestal) - Universidade Federal do Paraná, Curitiba, 1983.

YONEZAWA, J. T. A evolução da colheita mecanizada. Revista Opiniões, Ribeirão Preto, p. 17, jun./ago. 2010. 